\title{
The Impact of Robotics in the Economic of the Industrial Countries: Comprehensive Study
}

\author{
Nawal A Alonezi, Dr. Kawther A. Al-Dhlan* \\ engalrajh@gmail.com, K_aldhlan@hotmail.com
}

Department of Computer science and Information College of computer science and engineering, University of Ha'il

\begin{abstract}
Until the present time, man used to do everything by his arm and soul, from building, painting, transporting, installing, assembling, drilling, blowing, and exploration of minerals using the his capabilities, which takes a lot of effort and high cost with many labour forces to complete the work. However, with the industrial revolution and the invention of artificial intelligence which improves the automation and simulation the act of humans as well as enhancing other complicated process that would never done with human. By industrial robots the critical tasks will successfully achieved in some certain environment. Robotics adaption is changed the economy in interesting way which encourage to invest in them. The advantages of robotics for the industrial world including the accuracy, efficiency, time management, quality and efficacy in controlling tasks. This promising technology gave a positive impact on the economy leadership to adapt it in their industrial production. This paper aimed to investigate the impact of the industrial robotics in economy by comparing the use and growth level of robotics in different developed country, the statistical analysis was provided to support the comparison. In addition, the current paper considered the direct benefits and issues that human career. This research indicated that by the next decades, the investment of industrial robotics will motivate researchers and developers to investment more in this filed, meanwhile, the future of employment and job seekers will have new requirements; that may affect positively on the productively with considering the humanity factors .
\end{abstract}

Keywords - Robotics rise; Industries growth; Robotics applications; Artificial intelligence .

\section{Introduction}

Rapidly the development in technology have led to interest in automation and robotics. Where robotics contributed the development of humanity in a surprising way by making the heavy and light industries tasks more scalable via statistical report. It is known that the countries in the world are competing in innovation and development of industries in various fields.[2] Robotics and automation can hold the key of industrial companies' progress which enhanced to use new technologies for increasing productivity, reducing the error percentage and manage time-consuming. In fact, Robots are becoming present in more and more not just in factories also in business areas. They can work alongside with human workers or completely replacing them. A survey to business leaders reveal has been published that $24 \%$ of companies in US has already been applying AI in the work environment such as amazon warehouse to stock the company uses a variety of robotics that can handle out all the daily tasks related to items type from retrieving, checking, packaging and shipping customer items through the transportation that already designated for it. Tesla motors have the leadership also as the first company which develop electric cars that can drive without human interventions using an intelligent agent. The observation through survey shows expected increasing in percentage of using robots in the beginning of 2022 which exceeds up to $60 \%$. Robots usage are not limited in factories or 
companies but also widely used through applications that may be in hand such as smart devices based in digital voice assistants such as Siri or Alexa more than $62 \%$ used this application for reservation or shopping. Services robots sales continue growing at annual rates of greater than $30 \%$ according to the International Federation of Robotics (2018). In the next decades companies' visions are expansions the use of robotics services will increase to serve different purposes inside these companies which lead to create a professional work environment either for employees or customers through perform a public interaction that leads to growth in different sectors the global sales of service robots in 2017 divided the percentage in of robot sales in each sector (see Fig.1). Robotics can be also involved in medical fields they can act and interact to serve the doctors and patient as will. So, robotics can do operations with high accuracy and more quality according to the giving data. Recently, robots are even being used in therapy sessions to assessment the patient psychologically. Robot helping hand rise in a horizon in form of providing efficient services to elderly people they are programmed to understand the actions of an elderly person's habits they can help remind them about an appointment or even save a life if an elderly person falls it will automatically alert caregivers or emergency services. The current study provides a comprehensive literature review to investigate the impact of industrial robots on the internal incomes of some countries that implement this intelligent technology in a wide range and how this rises the exports.

Its known right now that robots' contributions are not limited, they involve in all the sectors of our daily life. The researchers adopt China as case study among other industrial countries in marketing. China was ranked as first in terms of exports in the world. The following questions were drawn to achieve the aim of the study :

- What is expected from robotic technologies to add in the medium and long term of the country incomes?

- Does the new rise of robotics impact negatively human opportunity in the labour markets or shorter and longer terms?

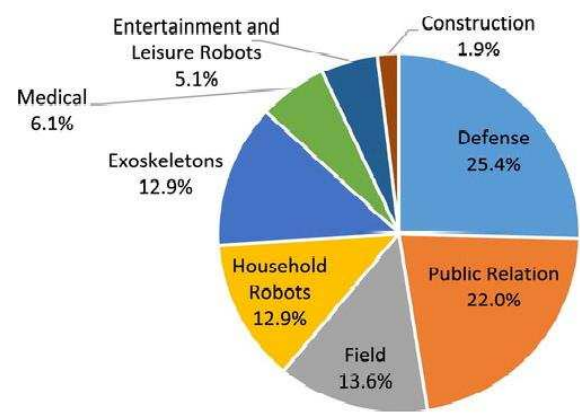

Fig. 1. Sectors of global sales of service robots in 2017 (excluding logistics); Source: IFR (2018).

\section{Research Methods}

The current study used a literary survey to collect data and information from different resources, with the consideration of the published resources, the impact factors of the reviewed articles and the governmental statistical. The collected information used to answer the research questions, and help to draw the conclusions.

\section{What drives robots to rise economy?}

The investing in robots is promising but not easy cause it depends in many important factors from programming, training, cost and the labour forces who will deal with these machines but all these obstacles 
could be prevented if they have good management. Rising of robots in economy depends on two issues, the increase in quality and efficiency more than anything else because they are scalable through production lines. Robots are widely used in big or small factories that are working in a large or small product in 2000 the rate of countries which installed robot's technology were divided in 6 main countries such as Korea, Taiwan, India, Brazil, and Poland. But who get the big share of the total stock from the shown statistical, is China indeed with more than fifth of the world's total stock of robots (see Fig. 2). China expands its leads in industrial robots up to $36 \%$ in 2017 which shows increasing in rate of global sales more than $30 \%$ in 2016 . The expected future statistical shows rising in the year of 2030 with approximately14 million industrial robots in use just in China. In contrast, China industrial robots use shows increasing since 2000 with more than 370,000 units than other countries such as US and Europe which shows fallen under $40 \%$ of the global share from its peak of close to $50 \%$ in 2009. Actually, robots rising increases productivity and competitiveness on the same scale, this particularly will be more effective for businesses with small to medium size companies represented the backbone of both developed and developing country economies. Without adopt rising of robots leads the large companies to increase their competitiveness through faster product development and delivery. On other hand the comparison with country such as Japan the leader in world of automation shows reduction in the stock activity of robots by around 100,000 units since the start of the millennium production, this leads Japan to replacing China's manufacturing and migration of many production facilities [9].

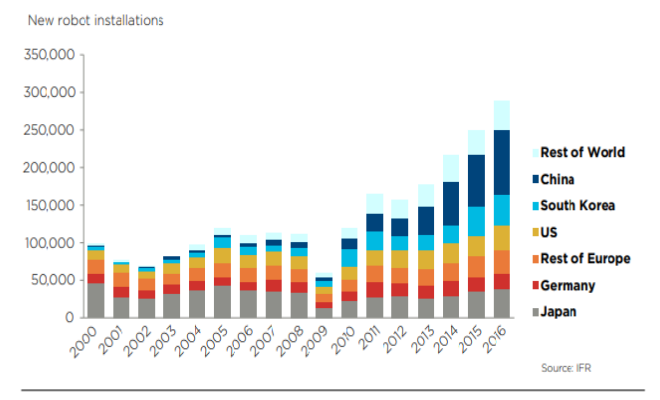

Fig. 2. Robot installations by country, 2000-2016; Source: IFR (2018).

\section{Industries driving growth}

Robots and automation of applications used in the industry to increase production line that will cover the need or keep the goods and services available whenever the need calls for it So, practically statistical study from the International Federation of Robotics shows the total incomes of five countries in 2018 which installing the robot's services in production line each country get incomes according to their export these countries show contradiction (refer to table 1). China farther ahead than other countries with nearly $30 \%$ more than others this shows the determination to advance industry productions through robotics used based on medium or long terms. This will take us to the future where robots and humans working side by side in factories or companies doing and achieving the same tasks and providing a service together. One of the most concerns in Robots use in labour market is not the high cost or the need of expertise but replacing a human by robot which can be programmed to achieve the activities that the humans can. The latest statistical show decreasing of labour hands with less than $10 \%$ of total jobs which can be fully automate. The net impact of robots on the work environment increasing though the quality of work is positive. On the other hand, the automation sector provides the opportunities for humans to focus on some important tasks inside these companies from higherskilled, higher-quality and higher-paid tasks. The International Federation of Robot recently believes that robot tax is unwarranted which means positively the improving impact of robotic investment on employment and wages will be needed in marketing. Undermining the competitiveness of companies and states. Leadership and 
decision makers in business need to assess the means and investments of generating revenues to cover social payments due to a large number of structural factors. These decisions will improve productivity, increase competitiveness, and create more jobs than just replacing them [8].

\section{Industrial Applications}

An industrial robot can be guided by external or internal input to deal with any work environment. These machines should be programmable to do complex tasks in a perfect way automatically with more precision, efficiency and capability.

A robot structure is designed to simulate the human ability through moving forwards or backwards, they are designed to movements in $2 \mathrm{D}$ or $3 \mathrm{D}$ depend on the mission they want to accomplish.[5]. The tasks the industrial robots are countless they can do everything depends in the location,

TABLE I. THE EXPROTS OF THE INDUSTRIAL COUNTRIES

\begin{tabular}{|c|l|l|}
\hline Rank & Markets & Incomes \\
\hline 1 & China & 39,351 \\
\hline 2 & Japan & 17,346 \\
\hline 3 & Germany & 15,673 \\
\hline 4 & U.S. & 15,246 \\
\hline 5 & South Korea & 11,034 \\
\hline
\end{tabular}

designing and programming. The robots can cover most tasks such as pick, drill, package, cutting operation, coating, assembling operation and much more.[3].The main types of industrial robots are six they can be classified as big machines they can be used in heavy industries such as Cartesian, SCARA, Cylindrical, Bin picking robotics and Medium payload handling robotic.

\section{Examples of Industrial Robot}

\subsection{Bin picking robotics:}

This type using 3D object recognition its can turned around the location production its divided into two parts one arranged and transferred by workers and the other parts stored in bins such as bolts, the worker can easily deal with this type inside the work site cause of its flexibility that can handle several types of parts in the same time as shown in Fig.3. The process to handle large parts in bins always requires complicated recognition cause this type is designed in $3 \mathrm{D}$ which sometimes required a lot of time in measuring data.

\subsection{Medium payload handling robotic:}

This type depends on automation cause its deal directly with medium-weight objects from $50 \mathrm{~kg}$ up to $200 \mathrm{~kg}$. The workers can handle it through movements or using the crane operation. Many production sites are expected to be automated because automation provides safety and secure which helped to increase the productivity. However, objects to be handled using this type of arms robot or other types for general large or heavy production the measurements and position should be previously specified cause without setting the measurements a delay in automation with robots developed a system that can load and unload engine blocks which cause overloaded up to $60 \mathrm{~kg}$ and off of pallets, and automatically transfer them to the inspection process when necessary(See Figure 4)In addition, the adoption of floating mechanism as a hand tool equipped for grasping a work piece from above enables smooth[2]. 


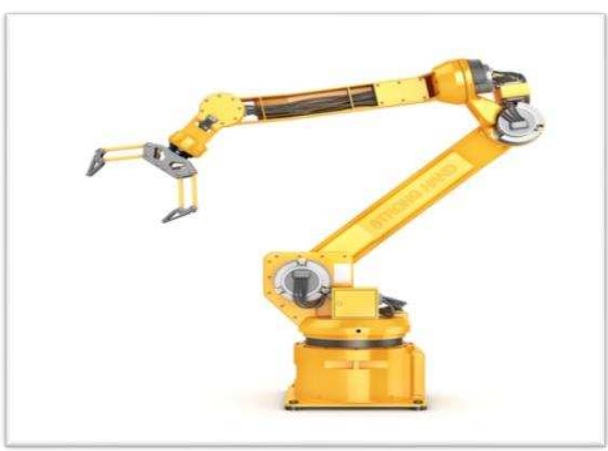

Fig. 3. Bin picking robotics; Source: Industrial Robot.

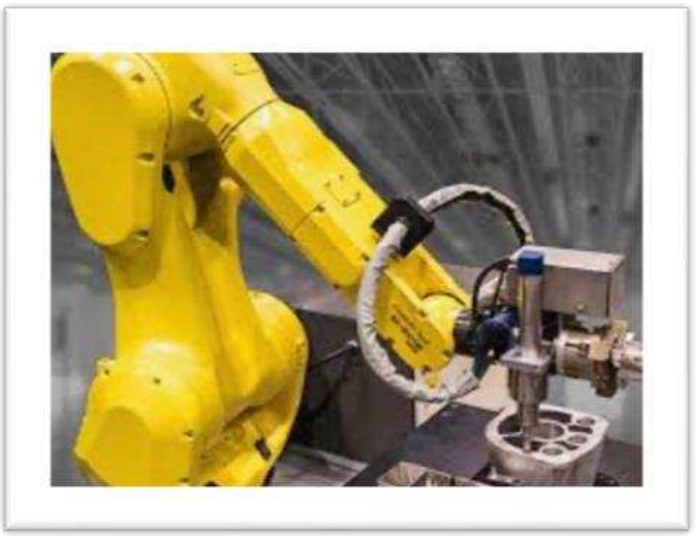

Fig. 4. Medium payload handling robotic, Source: Industrial Robot

TABLE2 IN ECONOMY.

SUMMARIZED THE POSITIVE AND NEGATIVE EFFECTS THAT RESULTED FROM UTILIZING ROBOTS

\begin{tabular}{|c|c|}
\hline Advantages & Disadvantages \\
\hline $\begin{array}{ll}\text { - } & \text { Robot take less time, in } \\
\text { - } & \text { Dompletion works . } \\
\text { - } & \text { heavy or light } \\
\text { - } & \text { Reducing the error rate. } \\
\text { - } & \text { High quality. } \\
\text { - } & \text { Providing operating cost. } \\
\text { - } & \text { Provide Capability by upgrading } \\
\text { - } & \text { payload. } \\
\text { - } & \text { Provide accuracy and more safety } \\
\text { - } & \text { Faster communication with } \\
& \text { external equipment. }\end{array}$ & $\begin{array}{l}\text { - High cost in maintenance } \\
\text { - Need to be trained. } \\
\text { - High cost of expertise } \\
\text { - impact of jobs for workers } \\
\text { by replacing them with } \\
\text { machines who can do their } \\
\text { work. }\end{array}$ \\
\hline
\end{tabular}




\section{Conclusion}

The repaid change of a countries economy that stands for manufacturing for small, medium, or long term according to supply and demand for local or global markets leads to real investments in the future for robots and automation. This increasingly shapes work environments for individual and government in higher levels and expectations. This supporting investment will also increase researches and developments in robotic used according to the points of view mention by the researcher in this paper. The robots used have shown improvements through statistical analysis and scales in production lines because of the high standards in quality, safety, accuracy, and efficiency, in these machines. Without forgetting to give attention to the labour market hands by providing skills and education for re-training existing, or future workers. This will reduce the concern about the future of employment and job seekers for individuals through automation that depends on expertise hands with high skills in programming. Without adopt robots, and automation leads to digital developments in the economy of countries for the next decades.

\section{References}

[1] Arkin, R. (2011). Military robotics and the robotics community s responsibility. Industrial Robot: An International Journal, 38(5)

[2] Crowe, S., 2020. Top 5 Countries Using Industrial Robots In 2018: IFR. [online] The Robot Report. Available at: $<$ https://www.therobotreport.com/top-5-countries-using-industrial-robots-2018/> [Accessed 13 December 2020].

[3] Hughes, J., Gilday, K., Scimeca, L., Garg, S. and Iida, F., 2019. Flexible, adaptive industrial assembly: driving innovation through competition. Intelligent Service Robotics, 13(1), pp.169-178.

[4] Papakostas, N., Michalos, G., Makris, S., Zouzias, D. and Chryssolouris, G., 2011. Industrial applications with cooperating robots for the flexible assembly. International Journal of Computer Integrated Manufacturing, 24(7), pp.650-660.

[5] Wegener, K., Chen, W., Dietrich, F., Dröder, K., \& Kara, S. (2015). Robot Assisted Disassembly for the Recycling of Electric Vehicle Batteries. Procedia CIRP, 29, 716-721.

[6] Mihret, E., 2020. Robotics and Artificial Intelligence. International Journal of Artificial Intelligence and Machine Learning, 10(2), pp.57-78.

[7] Pham, Q., Madhavan, R., Righetti, L., Smart, W., \& Chatila, R. (2018). The Impact of Robotics and Automation on Working Conditions and Employment [Ethical, Legal, and Societal Issues]. IEEE Robotics \& Automation Magazine, 25(2), $126-128$.

[8] How robots change the world. (2019). Economic Outlook, 43(3), 5-8.

[9] HARADA, K. (2018). Research Trends on Assembly Automation by Using Industrial Robots. Journal Of The Japan Society For Precision Engineering, 84(4), 299-302.

[10] Dottori, D. (2020). Robots and Employment: Evidence from Italy. SSRN Electronic Journal. doi: 10.2139/ssrn.3680743

[11] Belanche, D., Casaló, L., Flavián, C., \& Schepers, J. (2019). Service robot implementation: a theoretical framework and research agenda. The Service Industries Journal, 40(3-4), 203-225.

[12] TADOKORO, S. and UCHIZONO, T., 2017. Impact Tough Robotics Challenge. The Proceedings of JSME annual Conference on Robotics and Mechatronics (Robomec), 2017(0), pp.1P1-R01.

[13] Roboticstomorrow.com. 2020. Robotics and Automation Stories, Videos, Articles, Interviews, Reviews \& News | Roboticstomorrow. [online] Available at: 〈https://www.roboticstomorrow.com/> [Accessed 13 December 2020].

[14] Arduengo, M. and Sentis, L., 2020. The Robot Economy: Here It Comes. International Journal of Social Robotics.

[15] Thurbon, E. and Weiss, L., 2019. Economic statecraft at the frontier: Korea's drive for intelligent robotics. Review of International Political Economy, pp.1-25.

[16] Valente, A., 2016. Reconfigurable industrial robots: A stochastic programming approach for designing and assembling robotic arms. Robotics and Computer-Integrated Manufacturing, 41, pp.115-126 